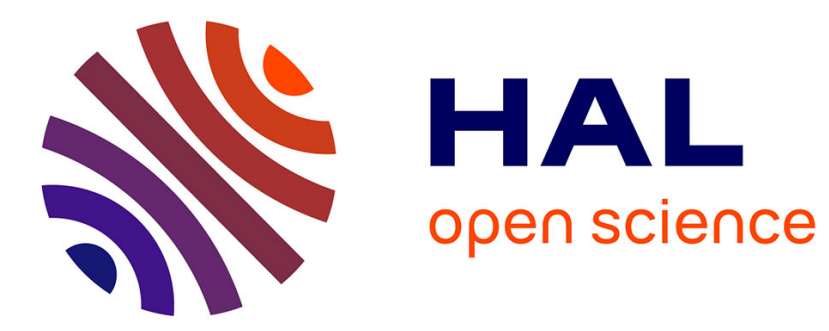

\title{
Workspace and cuspidality analysis of a 2-X planar manipulator
}

Matthieu Furet, Philippe Wenger

\section{To cite this version:}

Matthieu Furet, Philippe Wenger. Workspace and cuspidality analysis of a 2-X planar manipulator. 4th IFToMM Symposium on Mechanism Design for Robotics, Sep 2018, Udine, Italy. hal-01810820

\section{HAL Id: hal-01810820 \\ https://hal.science/hal-01810820}

Submitted on 8 Jun 2018

HAL is a multi-disciplinary open access archive for the deposit and dissemination of scientific research documents, whether they are published or not. The documents may come from teaching and research institutions in France or abroad, or from public or private research centers.
L'archive ouverte pluridisciplinaire HAL, est destinée au dépôt et à la diffusion de documents scientifiques de niveau recherche, publiés ou non, émanant des établissements d'enseignement et de recherche français ou étrangers, des laboratoires publics ou privés. 


\title{
Workspace and cuspidality analysis of a $2-\mathrm{X}$ planar manipulator
}

\author{
Matthieu Furet and Philippe Wenger \\ Laboratoire des Sciences du Numérique de Nantes (LS2N), CNRS, Ecole Centrale de \\ Nantes, 44321 Nantes, France
}

\begin{abstract}
This paper analyzes the workspace of a planar 2-X manipulator, i.e. made of two crossed four-bar mechanisms in series. This architecture has some advantages over classical 2-R manipulators such as its ability to be driven with tendons, but its kinematics is more challenging because of a variable instantaneous center of rotation of the Xmechanisms. The workspace boundaries are determined algebraically and its accessibility is analyzed. In the absence of joint limits, the workspace has regions with two and four inverse kinematic solutions. Depending on the values of its geometric parameters, the manipulator at hand may be cuspidal, i.e. it can change its posture without meeting a singularity. A necessary and sufficient condition is stated for the manipulator to be cuspidal. The effect of joint limits is analyzed and the accessibility regions are further classified according to the reachable configurations of each X-mechanism in these regions.
\end{abstract}

Keywords: Kinematics, crossed four-bar mechanism, Workspace, Cuspidal

\section{Introduction}

A crossed four-bar mechanism, referred to as X-mechanism, is a four-bar mechanism assembled in a X-shape configuration, see figure 1. Because of a variable instantaneous center of rotation (ICR), this mechanism has a large range of motion. Moreover, tendon driven actuation can be easily implemented and a lightweight manipulator with remote actuation can be designed by stacking several such mechanisms [1]. Eventually, lateral springs can be added on each side of the mechanism, thus defining a X-shape Snelson tensegrity mechanism [2], suitable for variable stiffness and natural interaction with the environment [3]. A tensegrity structure is an assembly of compressive elements (bars) and tensile elements (cables, springs) held together in equilibrium [4]. Tensegrity is known in architecture and art for more than a century [5] and is suitable for modeling living organisms [6]. Tensegrity mechanisms have been more recently studied for their promising properties in robotics such as low inertia, natural compliance and deployability [7],[8],[9]. This work is part of the AVINECK project involving biologists and roboticists with the main goal to model and design bird necks. Accordingly, a class of planar tensegrity manipulators made of a series assembly of 
several Snelson's X-shape mechanisms has been chosen as a suitable candidate for a preliminary planar model of a bird neck. Snelson's X-shape mechanisms have been studied by a number of researchers, either as a single mechanism [7],[9],[11] or assembled in series [12],[13],[3]. A planar two-degree-of-freedom manipulator is obtained with a series assembly of two such mechanisms. The manipulator can be driven with tendons threaded through the spring attachment points like in [3]. The detailed actuation scheme is not reported here. First investigations on the kinematics of such manipulators have proven more challenging than expected, in particular for the solution of the inverse kinematics [10]. This paper is a follow up of the work done in [10]. It focuses on the full workspace analysis of the manipulator and on cuspidality conditions. The workspace boundaries are determined algebraically and its accessibility is analyzed. In the absence of joint limits, the workspace has regions with two and four inverse kinematic solutions. Depending on the values of its geometric parameters, the manipulator at hand may be cuspidal, i.e. it can change its posture without meeting a singularity. A necessary and sufficient condition is stated for the manipulator to be cuspidal. The effect of joint limits is analyzed and the accessibility regions are further classified according to the reachable configuration of each X-mechanism in these regions.

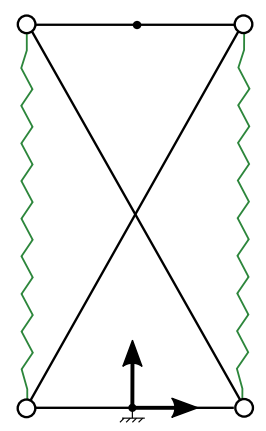

Fig. 1: Snelson's X-shape mechanism made of a crossed four-bar mechanism with lateral springs

\section{Manipulator modelling and Kinematic equations}

The manipulators studied consist of a series assembly of two identical X-mechanisms as shown in figure 2. Both the base bar and the upper bar are of length $b$ and the two crossed links are of length $L$ with $L>b$. A line segment of length $l_{i}$ is defined that links the middle points of the top and base bars of each mechanism $i$ (shown in red dotted line in figure 2). The angle between this line and the direction orthogonal to the base bar, referred to as $\theta_{i}$, is used to define the configuration of mechanism $i$ without ambiguity, assuming that it remains always in 
its crossed-bar assembly mode [10]. Accordingly, the manipulator configuration can be fully defined with $\left(\theta_{1}, \theta_{2}\right)$. To avoid any self collisions, the bars should be assembled in different layers or suitable joint limits should be defined. The base frame is centered at the middle point of the base bar of the first X-mechanism with the $\mathrm{X}$-axis aligned along this bar. The reference point $(x, y)$ is chosen as the middle point of the top-bar of the second X-mechanism (figure 2). Since the

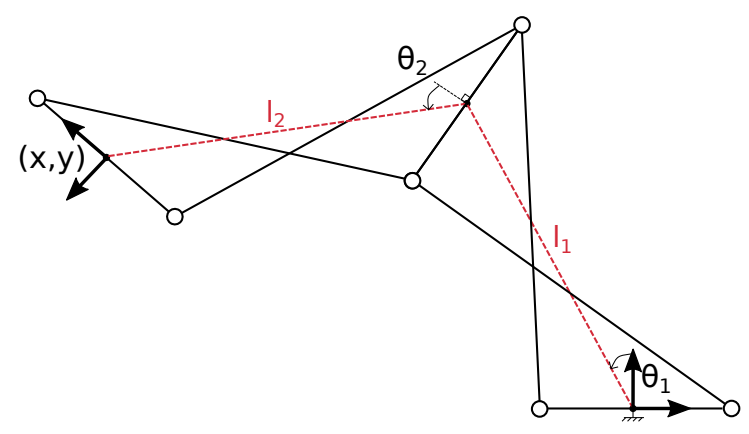

Fig. 2: Manipulator description

sides of each mechanism define an isosceles trapezoid, the length $l_{i}$ of the line segment that links the middle points of the top and base bars can be expressed as follows [10]:

$$
l_{i}\left(\theta_{i}\right)=\sqrt{L^{2}-b^{2} \cos ^{2}\left(\theta_{i}\right)}
$$

The direct kinematic equations of the 2 -X manipulator can be put in the following form :

$$
\left\{\begin{array}{l}
x=-l_{1}\left(\theta_{1}\right) \sin \left(\theta_{1}\right)-l_{2}\left(\theta_{2}\right) \sin \left(2 \theta_{1}+\theta_{2}\right) \\
y=l_{1}\left(\theta_{1}\right) \cos \left(\theta_{1}\right)+l_{2}\left(\theta_{2}\right) \cos \left(2 \theta_{1}+\theta_{2}\right)
\end{array}\right.
$$

where $l_{1}$ and $l_{2}$ are defined in (1). Note that these equations assume that each mechanism remains in its crossed-bar assembly-mode.

The inverse kinematics is much more challenging to establish and cannot be obtained from (2) easily. A methodology was proposed in [10], which makes it possible to derive a characteristic polynomial of degree four and it was shown that the manipulator may have up to four solutions.

\section{Workspace analysis}

The manipulator workspace is determined by means of its boundaries. These boundaries can be obtained from the discriminant of the 4th-order characteristic polynomial derived for the inverse kinematics. This characteristic polynomial in $t=\tan \left(\phi_{1} / 2\right)$ was derived in [10] and is recalled below, where $L$ has been set to 1 without loss of generality:

$$
a_{4} t^{4}+a_{3} t^{3}+a_{2} t^{2}+a_{1} t+a_{0}=0
$$


where :

$$
\begin{gathered}
a_{4}=(b+1)^{2}\left(b^{2} y^{2}+x^{4}+2 x^{2} y^{2}+y^{4}+4 x^{3}+4 x y^{2}+5 x^{2}+y^{2}+2 x\right) \\
a_{3}=4 y(b+1)\left(2 b^{2} x+b^{2}-2 x^{2}-2 y^{2}-4 x-1\right) \\
a_{2}=2\left(b^{4} y^{2}+b^{2} x^{4}+2 b^{2} x^{2} y^{2}+b^{2} y^{4}+b^{2} x^{2}-10 b^{2} y^{2}+x^{4}+2 x^{2} y^{2}+y^{4}-3 x^{2}+9 y^{2}\right) \\
a_{1}=4 y(b-1)\left(2 b^{2} x-b^{2}+2 x^{2}+2 y^{2}-4 x+1\right) \\
a_{0}=(b-1)^{2}\left(b^{2} y^{2}+x^{4}+2 x^{2} y^{2}+y^{4}-4 x^{3}-4 x y^{2}+5 x^{2}+y^{2}-2 x\right)
\end{gathered}
$$

The discriminant of this polynomial is derived with the help of a symbolic computing software. Accordingly, a polynomial equation of degree 16 in $x$ and $y$ is obtained. This equation is much too large to be displayed here and can be found in [15]. Figure 3 shows the plot of these boundary curves for three cases. In the second and third cases, they divide the workspace into three regions. In the largest one, the manipulator admits two inverse kinematic solutions. In the two smaller regions (filled in grey), there are four solutions. Figure 4 shows the four inverse kinematic solutions for the manipulator defined by $L=1$ and $b=9 / 10$, at $x=0, y=1$.
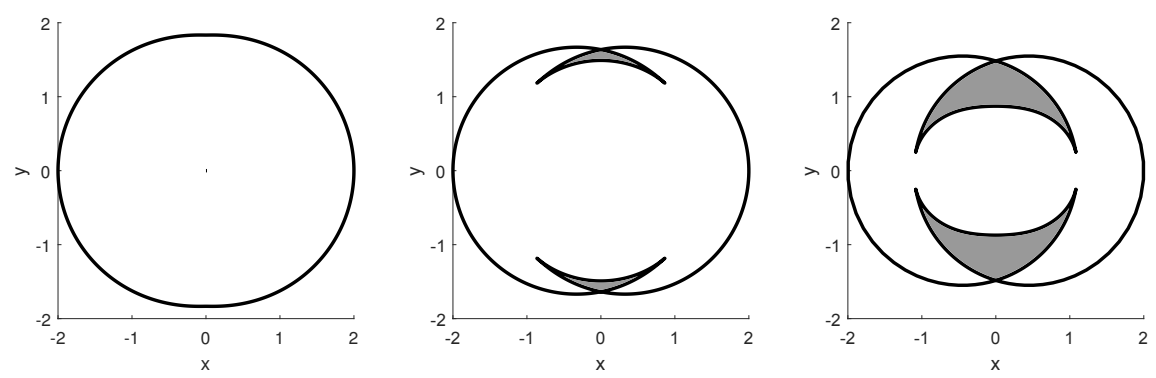

Fig. 3: Workspace boundaries when $L=1$ and $b=2 / 5$ (left), $b=2 / 3$ (center), $b=9 / 10$ (right)
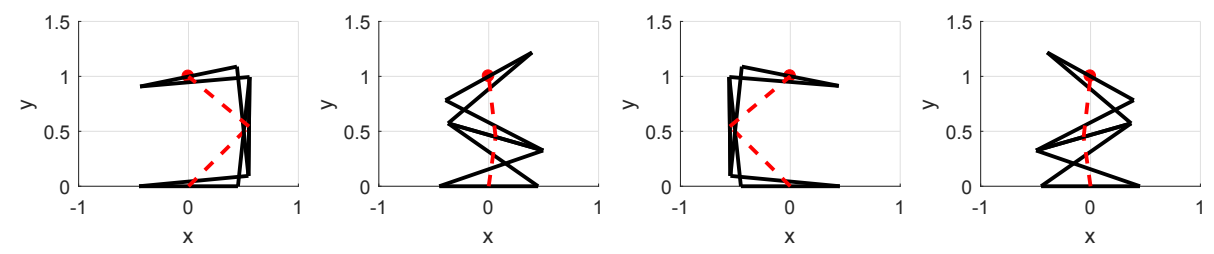

Fig. 4: The four inverse solutions at $x=0$ and $y=1(L=1$ and $b=9 / 10)$ 
The workspace boundaries studied so far are associated with the singularities of the 2-X manipulator as a whole where two inverse kinematic solutions coincide. These singularities occur when the ICRs of the two X-mechanisms and the output point are aligned [10]. On the other hand, each X-mechanism has its own singularities, i.e. when it is completely flat. They are defined by $\theta_{i}= \pm \frac{\pi}{2}$. The role of the X-mechanism singularities is to distinguish mechanism solutions in a configuration where the upper bar is above the base bar from those where the upper bar is below the base bar. These two configurations, referred to as configuration up and configuration down, respectively, are illustrated in figure 5.

The curves associated with the singularities of the X-mechanisms are now plotted in the workspace, see figure 6 . The additional curves divide the 2 -solution and the 4-solution regions into smaller regions associated with different combinations of mechanism configurations as explained in figure 6 . For example, the red region with both $\|$ and // lines means that two solutions are of the type $M_{i}$ (both mechanisms are up) and the other two are $M_{1} \overline{M_{2}}$ (the first mechanism is up and the second is down). In the context of a tensegrity manipulator driven with cables along the sides of the mechanisms, the reachable workspace should be reduced to those regions where at least one solution exists with both mechanisms up, namely, those regions that have vertical lines in figure 6. Note that there exists a small region where the four solutions have the two mechanisms in configuration up (the red diamond upper region with only || lines in the zoomed-in view).
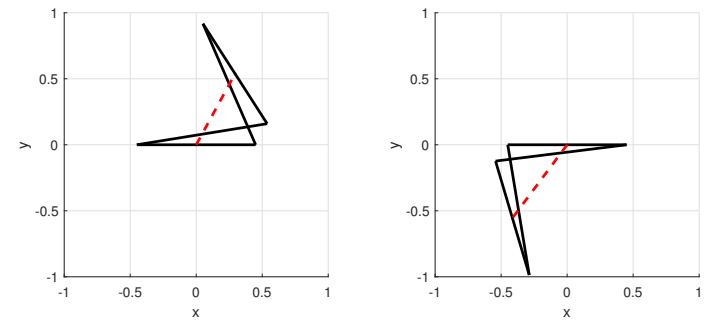

Fig. 5: X-mechanism in configuration up (left) and down (right)

\section{Influence of the geometric parameters and cuspidality condition}

The workspace may have four-solutions regions. The boundaries of the two 4solution regions have three singular points: a node and two cusps. The existence of cusps confirms the fact that the manipulator is cuspidal. A non-singular solution changing motion can be defined by encircling one of the cusps [14], [16].

From figure 3 , the 4 -solution regions are smaller when $b$ is smaller. In fact, the four-solution regions may even vanish (figure 3 , left) and so do also the 
cusp points. The transition, called swallowtail bifurcation [17], occurs when the two cusps and the node coincide and define a point with four coincident inverse kinematic solutions. Thus, a geometric condition for having two cusps and four inverse solutions can be established by deriving a condition on the geometric parameters for the 4th-order characteristic polynomial to admit a quadruple root. The latter condition can be stated as follows [18]:

$$
\left\{\begin{array}{l}
a_{4} a_{0}-\frac{1}{4} a_{3} a_{1}+\frac{1}{12} a_{2}^{2}=0 \\
\frac{1}{6} a_{4} a_{2} a_{0}-\frac{1}{16} a_{4} a_{1}^{2}-\frac{1}{16} a_{3}^{2} a_{0}+\frac{1}{48} a_{3} a_{2} a_{1}-\frac{1}{216} a_{2}^{3}=0 \\
\frac{1}{6} a_{4} a_{2}-\frac{1}{16} a_{3}^{2}=0
\end{array}\right.
$$

The above system can be simplified by observing that the quadruple root should appear at $x=0$ due to symmetry. Solving $b$ from the last two equations in (9) yields $b=\sqrt{6} / 6$. When $b>\sqrt{6} / 6$, the manipulator is cuspidal and has four inverse solutions, otherwise it has only two solutions and it is noncuspidal. Keeping in mind that $L$ was set to 1 and taking into account the assembly condition, the condition is actually $b<L<b \sqrt{6}$. the following theorem can thus be stated as follows:

Theorem: a planar 2-dof manipulator made of two identical symmetrical Xmechanisms with crossed link lengths $L$ and base link $b$ is cuspidal and has four inverse kinematic solutions if and only if $b<L<b \sqrt{6}$.

This theorem is useful for the designer as well as for control.

\section{Influence of the joint limits}

Unlimited joints were assumed so far. Figure 7 shows an example with $-\pi / 3<$ $\theta_{1}<\pi / 3$ and $-\pi / 3<\theta_{2}<\pi / 3$, and the geometric parameters $L=1, b=2 / 3$. As compared with figure 6 , the curves associated with the flat configurations have been replaced by curves associated with the joint limits and a great part of the manipulator singularity curves have disappeared. There are two solutions in the region filled in grey and only one solution in the two white regions. Moreover, it can be shown that all solutions are only reachable with both mechanisms in configuration up due to the joint limits.

\section{Conclusion}

The workspace of a planar manipulator made of two X-mechanisms in series has been studied. The manipulator was shown to have two or four solutions depending on its geometric parameter values. When it has four solutions, the manipulator turns out to be cuspidal. The influence of the geometric parameters on the shape and size of the workspace was analyzed and a geometric condition for the manipulator to be cuspidal was established. The influence of joint limits was also analyzed. Internal collisions were not considered since a suitable design by assembling the rods in different planes allows avoiding any physical 


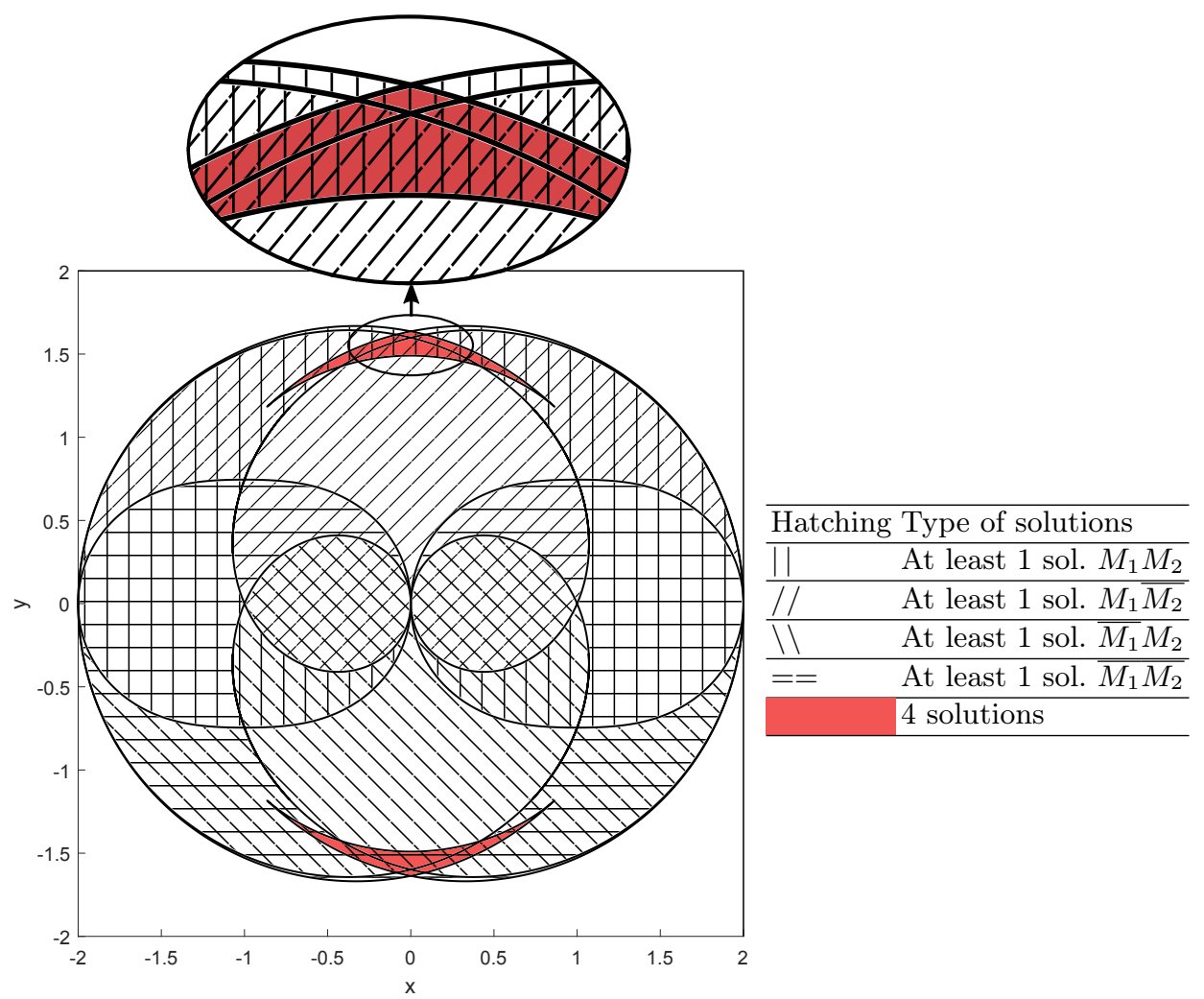

Fig. 6: Workspace regions with different numbers and types of solutions $(L=1$, $b=2 / 3 . \overline{M_{i}}$ (resp. $M_{i}$ ) means that mechanism $i$ is in configuration down (resp. up)

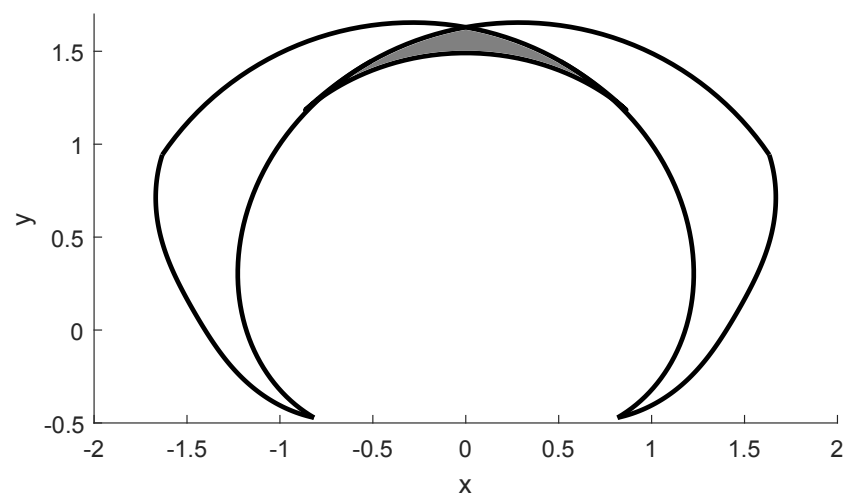

Fig. 7: Workspace boundaries when $-\pi / 3<\theta_{1}<\pi / 3$ and $-\pi / 3<\theta_{2}<\pi / 3$ $(L=1, b=2 / 3)$. The region filled in grey (resp. white) has two (resp. 1) solutions 
interference. In future work, different actuation strategies will be considered and compared with each other.

Acknowledgement This work has been conducted in part with the support of the French National Research Agency (AVINECK Project ANR-16-CE33-0025).

\section{References}

1. K. Moored et al., Analytical predictions, optimization, and design of a tensegritybased artificial pectoral fin, Int. J. of Solids and Structures, Vol. 48, pp3142-3159, 2011

2. K. Snelson, Continuous Tension, Discontinuous Compression Structures, US Patent No. 3,169,611, 1965

3. D. L Bakker et al., Design of an environmentally interactive continuum manipulator, Proc.14th World Congress in Mechanism and Machine Science, IFToMM'2015, Taipei, Taiwan, 2015

4. R. B. Fuller, Tensile-integrity structures, United States Patent 3063521,1962

5. Skelton, R. and de Oliveira, M., Tensegrity Systems. Springer, 2009

6. S. Levin, The tensegrity-truss as a model for spinal mechanics: biotensegrity, J. of Mechanics in Medicine and Biology, Vol. 2(3), 2002

7. M. Arsenault and C. M. Gosselin, Kinematic, static and dynamic analysis of a planar 2-dof tensegrity mechanism, Mech. and Mach. Theory, Vol. 41(9), 1072-1089, 2006

8. C. Crane et al., Kinematic analysis of a planar tensegrity mechanism with presstressed springs, in Advances in Robot Kinematics: analysis and design, pp 419-427, J. Lenarcic and P. Wenger (Eds), Springer (2008)

9. P. Wenger and D. Chablat, Kinetostatic Analysis and Solution Classification of a Planar Tensegrity Mechanism, proc. 7th. Int. Workshop on Comp. Kinematics, Springer, ISBN 978-3-319-60867-9, pp422-431, 2017.

10. M. Furet et al., Kinematic analysis of planar 2-X tensegrity manipulators, Proc. Advances in Robot Kinematics, Bologna, Italy, June 2018.

11. Q. Boehler et al., Definition and computation of tensegrity mechanism workspace, ASME J. of Mechanisms and Robotics, Vol 7(4), 2015

12. JB Aldrich and RE Skelton, Time-energy optimal control of hyper-actuated mechanical systems with geometric path constraints, in 44th IEEE Conference on Decision and Control, pp 8246-8253, 2005

13. S. Chen and M. Arsenault, Analytical Computation of the Actuator and Cartesian Workspace Boundaries for a Planar 2-Degree-of-Freedom Translational Tensegrity Mechanism, Journal of Mech. and Rob., Vol. 4, 2012

14. J. El Omri and P. Wenger, How to recognize simply a non-singular posture changing manipulator, Proc. 7th Int. Conf. on Advanced Robotics, 215-222, 1995

15. M. Furet and P. Wenger, Derivation of a polynomial equation for the boundaries of 2-X manipulators, Technical report, LS2N, April 2018.

16. P. Wenger, Cuspidal and noncuspidal robot manipulators. Special issue of Robotica on Geometry in Robotics and Sensing, Volume 25(6), pp.677-690, 2007

17. F. Thomas and P. Wenger, , On the Topological Characterization of Robot Singularity Loci. A Catastrophe-Theoretic Approach, IEEE International Conference on Robotics and Automation ICRA 2011, 9-13 mai 2011, Shanghai.

18. P. Wenger, D. Chablat, M. Baili, A DH parameter based condition for 3R orthogonal manipulators to have four distinct inverse kinematic solutions, ASME Journal of Mechanical design, Vol. 127(1), pp 150-155, 2005 